Check for updates

Cite this: RSC Adv., 2017, 7, 33413

\section{Multi-type quantum dots photo-induced doping enhanced graphene/semiconductor solar cell}

\author{
Jianghong Wu, ${ }^{a}$ Sirui Feng, ${ }^{a}$ Zhiqian Wu, ${ }^{a}$ Yanghua $\mathrm{Lu}^{\mathrm{a}}$ and Shisheng Lin (D) *ab \\ A viable approach to enhance the photovoltaic performance of graphene (Gr)/semiconductor solar cells \\ has been demonstrated. In order to take full advantage of the solar energy in the range of visible and \\ ultraviolet light, InP and $\mathrm{ZnO}$ quantum dots (QDs) with band gaps of $2.4 \mathrm{eV}$ and $3.3 \mathrm{eV}$, respectively, are \\ simultaneously introduced to dope $\mathrm{Gr}$ by a photo-induced doping mechanism. Raman and \\ photoluminescence measurements indicate that the photo-induced holes diffuse into Gr, leading to $\mathrm{p}$ - \\ doping of Gr. As a result, the power conversion efficiency (PCE) of the Gr/GaAs heterostructure solar cell \\ with good stability can be improved from $8.57 \%$ to $11.50 \%$. Although this process is simple and feasible, \\ we emphasize that the mixed semiconductor QDs enhanced $\mathrm{Gr} /$ semiconductor heterostructure solar \\ cell is similar to the band gap engineering of traditional multi-junction bulk semiconductor solar cells.
}

Received 19th May 2017

Accepted 26th June 2017

DOI: $10.1039 / \mathrm{c} 7 \mathrm{ra05646j}$

rsc.li/rsc-advances interest in the photo-induced doping of Gr, which can improve the properties of two-dimensional optoelectronic devices based on Gr. ${ }^{27,28}$ As shown in Fig. 1(a), the valence and conduction bands of semiconductor clearly bend when Gr contacts with the semiconductor. Actually, the depletion layer of the semiconductor is close to the interface of heterojunction, namely the surface. Simultaneously, the screening length in Gr is around $0.6 \mathrm{~nm}$ and the van der waals bonding nature, ${ }^{29}$ so the Fermi level of Gr can be adjusted by photo-induced doping. As for incident light, the ultraviolet and visible photons can penetrate into the $\mathrm{Gr} / \mathrm{semiconductor} \mathrm{heterostructure} \mathrm{for} \mathrm{a} \mathrm{long} \mathrm{distance}$

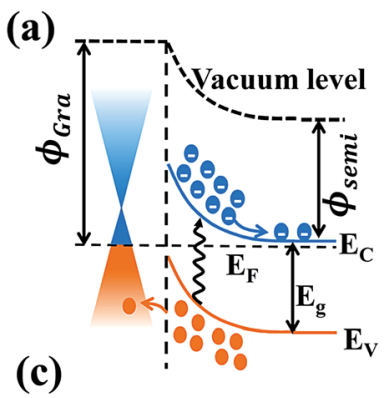

(b) isfactory. Distinct from a traditional PN junction, some novel device structures have been developed for achieving high performance solar cells. ${ }^{\mathbf{1 0 - 1 2}}$ Under this context, the solar cells based on the $\mathrm{Gr} /$ semiconductor heterostructure have profoundly drawn the attention of scientific community, ${ }^{13}$ because Gr possesses a few outstanding electrical and optical properties, containing extraordinarily high carrier mobility, ${ }^{\mathbf{1 4}}$ $2.3 \%$ constant absorption of visible light,${ }^{15}$ micro-scale ballistic transport, ${ }^{16}$ abnormal quantum Hall effect, ${ }^{17}$ outstanding thermal conductivity ${ }^{\mathbf{1 8}}$ and high mechanical strength. ${ }^{19}$

It has been reported that the electrical and optical properties of $\mathrm{Gr}$ can be easily adjusted by surface adsorption of foreign molecules, ${ }^{20,21}$ electrical gating, ${ }^{22}$ magnetic effects, ${ }^{23,24}$ and surface plasmons. ${ }^{25,26}$ Especially, there is a continuously rising

${ }^{a}$ College of Microelectronics, College of Information Science and Electronic Engineering, Zhejiang University, Hangzhou, 310027, China. E-mail: shishenglin@ zju.edu.cn; Tel: +86-0571-87951555

${ }^{b}$ State Key Laboratory of Modern Optical Instrumentation, Zhejiang University, Hangzhou, 310027, China
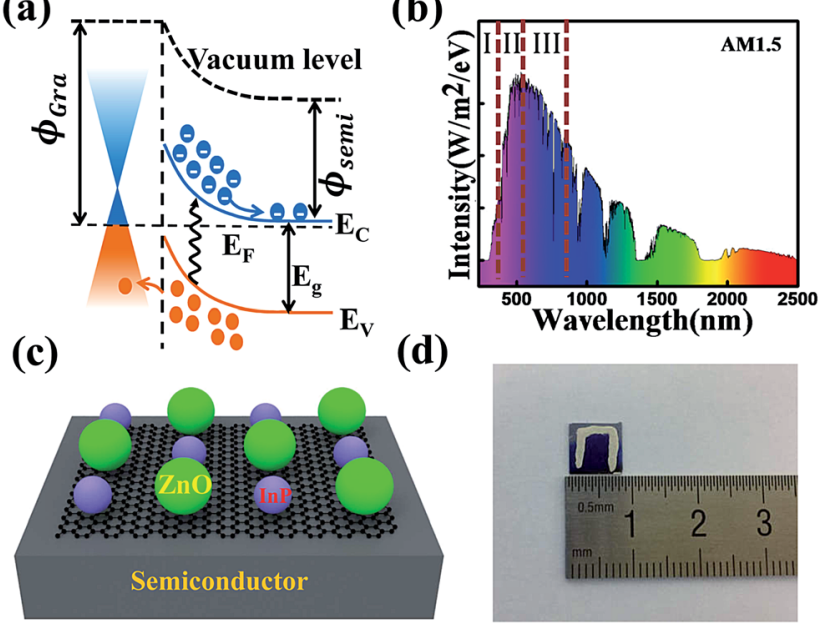

Fig. 1 (a) Physical illustration of depletion layer in Gr/semiconductor heterostructure. (b) AM1.5 solar spectrum with obvious minimal values thanks to molecular absorption in Earth's atmosphere. (c) Schematic structure of QDs/Gr/semiconductor. (d) Photograph of a QDs/Gr/ GaAs solar cell. 
and the photo-induced carriers cannot effectively be collected by the Gr and semiconductor, respectively. Therefore, it can be attractive to absorb the ultraviolet and visible light just on the surface of $\mathrm{Gr} /$ semiconductor solar cells, which can effectively dope Gr and lift up the barrier height of the heterostructure, leading to an obvious improvement of light conversion efficiency. As a result, it is reasonable to design a more effective photo-induced doping layer of Gr for usage of high efficient $\mathrm{Gr}$ / semiconductor solar cells. We come to the idea of using semiconductor QDs with varies of band gap as the photo-induced doping source. As shown in Fig. 1(b), the solar spectrum with a lot minimal values because of gas molecular absorption such as oxygen and carbon dioxide in the atmosphere. Generally, the wavelength of maximal energy intensity is about $500 \mathrm{~nm}$ which is close to the absorption peak of InP QDs and the external quantum efficiency (EQE) of Gr/GaAs solar cell is smaller in the ultraviolet band. ${ }^{22}$ In view of above-mentioned reasons, a synergetic photo-induced doping to enhance Gr/GaAs heterostructure solar cell is present in this work, which can utilize incident light more effectively. As shown in Fig. 1(c), ZnO QDs can absorb ultraviolet light and InP QDs absorb visible light whose wavelength is particularly below $530 \mathrm{~nm}$, respectively, generating excess holes injects into Gr. At the same time, thin film n-type GaAs can effectively absorb incident light whose wavelength is smaller than $874 \mathrm{~nm}$. Therefore, under this cooperative photo-induced doping, the final PCE can be improved by $34.2 \%$ in this work.

\section{Experimental section}

\subsection{Fabrication of solar cell}

Growth of monolayer $\mathrm{Gr}$ on copper foils was carried out at $1000{ }^{\circ} \mathrm{C}$ for $60 \mathrm{~min}$ with reaction source flux ratio of $\mathrm{CH}_{4}: \mathrm{H}_{2}$ equaling to $5: 1$ by chemical vapor deposition (CVD) technique. ${ }^{30}$ Then polymethyl methacrylate (PMMA) working as the supporting layer was spun on Gr before removing the copper foil, and PMMA was subsequently removed by acetone for about 10 min. Trilayer Gr was obtained by multi-transferring monolayer Gr. The obtained ZnO and InP QDs were eventually diluted in ethanol and cyclohexane solvent, respectively. Thin film ndoped GaAs with a thickness of $2 \mu \mathrm{m}$ was grown with metal organic CVD at $400{ }^{\circ} \mathrm{C}$, at the same time, the doping concentration of the GaAs wafer was about $1 \times 10^{18} \mathrm{~cm}^{-3}$. Then rear Au contact with a thickness of $60 \mathrm{~nm}$ was thermally evaporated on back surface of GaAs wafer. Native oxide on GaAs was removed by dipping the wafers into $10 \%$ wt dilute hydrochloric acid solution for $3 \mathrm{~min}$. Surface passivation of GaAs was achieved by soaking treatment of mixed solution of ammonia sulfide and ammonia for $10 \mathrm{~min}$. Gr was transferred onto the surface of ready GaAs, drying in air for half an hour and annealing for 10 min at $120^{\circ} \mathrm{C}$. Then PMMA at the surface of Gr was removed by acetone and silver paste was pasted onto $\mathrm{Gr}$, followed by 120 ${ }^{\circ} \mathrm{C} / 5$ min post-anneal. Basing on the above operation, Gr/GaAs solar cell preliminarily came into being. Finally, the key manipulation was carried out as follows: InP QDs were spun on $\mathrm{Gr}$ at $4000 \mathrm{rpm}$ for $60 \mathrm{~s}$, and then the similar operation was performed again so as to introduce ZnO QDs to the surface of Gr.

\subsection{Characterization}

InP and $\mathrm{ZnO}$ QDs were transferred to the carbon transmission electron microscopy (TEM) grid for morphology measurement using TEM (Tecnai F-30 operating at $300 \mathrm{kV}$ ). Trilayer Gr with and without QDs was characterized by Raman spectroscopy (Renishaw in Via Reflex) with $532 \mathrm{~nm}$ excitation source and 0.65 $\mathrm{mW}$ laser power. The absorption spectra and photoluminescence (PL) measurements of InP and $\mathrm{ZnO}$ QDs in ethanol and cyclohexane solvent were characterized by Cary 4000 of Agilent and FS920 of Edinburgh, respectively. PL measurements used to evaluate the charge transferring behaviors at the interface of Gr/QDs were characterized by a Jobin Yvon HR 640 monochromator with a $325 \mathrm{~nm} \mathrm{He-Cd} \mathrm{laser} \mathrm{as} \mathrm{the}$ excitation source. The photovoltaic performance of the QDs/Gr/ GaAs solar cells was tested with a solar simulator under AM1.5G condition at illumination intensity of $100 \mathrm{~mW} \mathrm{~cm}^{-2}$. The illumination intensity was calibrated with a standard Si solar cell. The current density-voltage $(J-V)$ data were recorded using Agilent B1500A system.

\section{Results and discussion}

InP and ZnO QDs are transferred to carbon grid for TEM characterizations to reveal the morphology and lattice structure of QDs. Fig. 2 shows TEM images of these two kinds of QDs in different magnifications and their electron diffraction pattern. Fig. 2(a) and (c) show the low-magnification TEM images of InP and ZnO QDs. From the high-magnification TEM images shown in Fig. 2(b) and (d), we can draw a conclusion that these two kinds of QDs possess a high crystal quality and average diameters of InP and ZnO QDs are about $5 \mathrm{~nm}$ and $15 \mathrm{~nm}$, respectively. Moreover, the insets show electron diffraction pattern of (a)

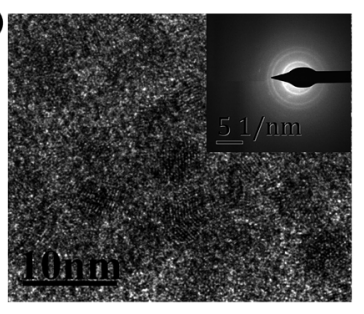

(c)

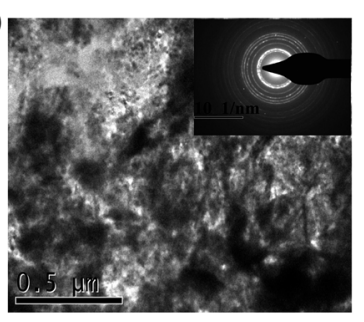

(b)

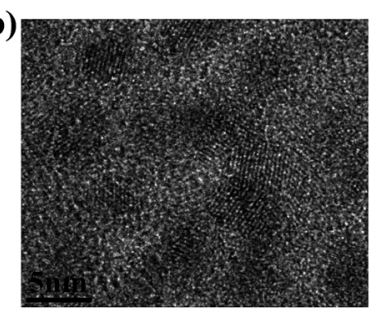

(d)

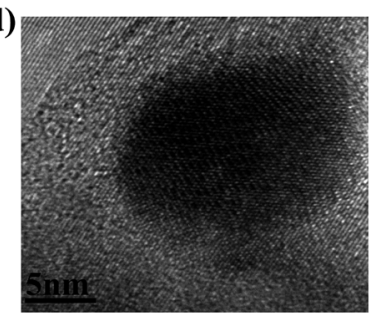

Fig. 2 Low-magnification (a) and high-magnification (b) TEM images of the InP QDs. Low-magnification (c) and high-magnification (d) TEM image of the $\mathrm{ZnO} Q \mathrm{QDs}$. Insets show electron diffraction pattern of the $\operatorname{lnP}(\mathrm{a})$ and $\mathrm{ZnO}$ (c) QDs. 
InP and ZnO QDs, where the diffraction lines can be well labeled by the lattice planes of InP and $\mathrm{ZnO}$. Trilayer Gr for Raman measurements is transferred to $\mathrm{SiO}_{2} / \mathrm{Si}$ substrate by wet transferring process, which is then coated with InP and $\mathrm{ZnO}$ QDs step by step. The corresponding Raman spectra of Gr are shown in Fig. 3(a). For as-grown Gr, there are three characteristic peaks locating at $1350 \mathrm{~cm}^{-1}, 1591 \mathrm{~cm}^{-1}$ and $2695 \mathrm{~cm}^{-1}$, corresponding to $\mathrm{D}, \mathrm{G}$ and $2 \mathrm{D}$ peaks, respectively. After spinning these two kinds of QDs on the surface of Gr, the G peak blue-shifts to $1596 \mathrm{~cm}^{-1}$, which indicates that p-doping of $\mathrm{Gr}$ is enhanced by coating InP QDs and ZnO QDs subsequently. Fig. 3(b) shows the absorption spectra of ZnO and InP QDs in ethanol and cyclohexane solvent, respectively. For InP QDs, absorbance at short wavelength is very high, while it is very weak at long wavelength, and the absorption peak locates around $485 \mathrm{~nm}$. For ZnO QDs, the absorbance is even weaker than InP QDs and the absorption peak locates around $374 \mathrm{~nm}$ of ultraviolet wavelength. Actually, these measurements are consistent with their inherent band gap $\left(E_{\mathrm{g}}\right)$ structure. To be specific, InP QDs with an even smaller $E_{\mathrm{g}}$ than ZnO QDs can absorb much more energy of incident light. Moreover, these two different kinds of QDs can absorb photon with different energy and their photo-inducing effect can be accumulated. At the same time, these two kinds of QDs possess weak absorbance in visible wavelength, so GaAs substrate can effectively absorb other available incident light.

Fig. 4(a) shows the PL spectra of InP and ZnO QDs in ethanol and cyclohexane solvent with the excitation laser line of 405 and $325 \mathrm{~nm}$, respectively. For InP QDs, there is a visible luminescence (VL) band with a maximum at about $515 \mathrm{~nm}$ which corresponds to the band-to-band recombination $(2.4 \mathrm{eV})$. For ZnO QDs, PL spectrum mainly contains an ultraviolet luminescence band with a maximum at about $380 \mathrm{~nm}$ and a VL band with a maximum at about $510 \mathrm{~nm}$. Indeed, the phenomenon of wide PL band probably attributes to the function of defect levels. Fig. 4(b) illustrates the PL spectra of the InP QDs with $325 \mathrm{~nm}$ excitation laser. It is notable that InP QDs are directly spun on $\mathrm{SiO}_{2} / \mathrm{Si}$ and $\mathrm{Gr} / \mathrm{SiO}_{2} / \mathrm{Si}$ substrate for PL measurements. In vertical direction, the PL intensity of InP QDs is one magnitude larger without Gr underlying than that of the case with $\mathrm{Gr}$ underlying, which demonstrates that a majority of laser- induced carriers hop into the Gr. Fig. 4(c) shows the PL spectra of $\mathrm{ZnO}$ QDs with $325 \mathrm{~nm}$ excitation laser on $\mathrm{SiO}_{2} / \mathrm{Si}$ and $\mathrm{Gr} / \mathrm{SiO}_{2} / \mathrm{Si}$ substrate. Similar experimental results can be distinctly observed that PL intensity reduces an order of magnitude after coating the QDs on the surface of Gr. Fig. 4(d) schematically illustrates the mechanism of the InP and $\mathrm{ZnO}$ QDs introducing photo-induced p-doping into Gr. More concretely, the photo-induced holes from valance band and defect levels hop into Gr, while electrons are trapped in the inner of QDs. Furthermore, the injected holes and negativecharged QDs can greatly influence the carrier concentration in $\mathrm{Gr}$, slightly adjusting the $E_{\mathrm{F}-\mathrm{Gr}}{ }^{31}$

The schematic structure of enhanced Gr/GaAs heterostructure solar cell with ZnO and InP ODs is shown in Fig. 5(a), which consists of $\mathrm{Au}$ gate electrode, n-type GaAs substrate, trilayer $\mathrm{Gr}, \mathrm{Ag}$ contact, $\mathrm{ZnO}$ and InP QDs. InP and ZnO QDS solution are dropped onto the surface of $\mathrm{Gr} / \mathrm{GaAs}$ solar cell and gently baked for $3 \mathrm{~min}$. $J-V$ curves of the $\mathrm{Gr} / \mathrm{GaAs}$ solar cell with and without QDs under AM1.5G illumination are shown in Fig. 5(b). Without any QDs, the best PCE $\left(\mathrm{PCE}=\frac{V_{\mathrm{oc}} \times J_{\mathrm{sc}} \times \mathrm{FF}}{P_{\mathrm{in}}} \times 100 \%\right.$. $)$ of device is $8.57 \%$ and the corresponding parameters of solar cell containing open circuit voltage $\left(V_{\mathrm{oc}}\right)$, short circuit current density $\left(J_{\mathrm{sc}}\right)$, circuit and fill factor (FF) are $0.67 \mathrm{~V}, 17.80 \mathrm{~mA} \mathrm{~cm}^{-2}$, and $71.80 \%$, respectively. After coating the InP QDs on surface of Gr at the front top of solar cell, $J_{\mathrm{sc}}$ is increased to $22.02 \mathrm{~mA} \mathrm{~cm}^{-2}, V_{\mathrm{oc}}$ is slightly increased to $0.68 \mathrm{~V}$, but FF is slightly decreased to $69.42 \%$, so the PCE is evidently increased to $10.39 \%$. To our best knowledge, this decreasing trend of FF primarily results from corresponding enhancement of Gr's surface resistance. Then some $\mathrm{ZnO}$ QDs are spun on the surface of $\mathrm{Gr}, J_{\mathrm{sc}}$ is further increased to $24.41 \mathrm{~mA} \mathrm{~cm}{ }^{-2}$, resulting in the finial PCE of $11.50 \%$. Therefore, the PCE enhancement resulting from the photoinduced doping is distinct. In Fig. 5(c), $J-V$ curves of the $\mathrm{Gr} /$ GaAs solar cells with and without QDs in the dark perform similar rectifying characteristics, from which ideality factor can be obtained through single-diode fitting based on the following eqn (1):

$$
J=J_{0}\left[\exp \left(\frac{q V}{N_{\mathrm{IF}} K T}\right)-1\right]
$$
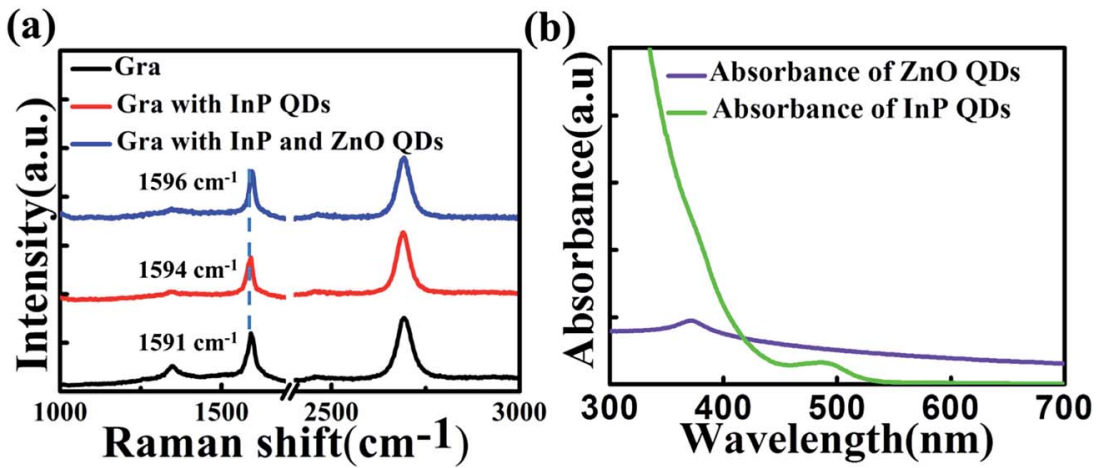

Fig. 3 (a) Raman spectra of trilayer $\mathrm{Gr}$ without and with QDs underlying on $\mathrm{SiO}_{2} / \mathrm{Si}$ substrate. (b) Absorption spectra of InP and $\mathrm{ZnO}$ QDs in corresponding solvent. 

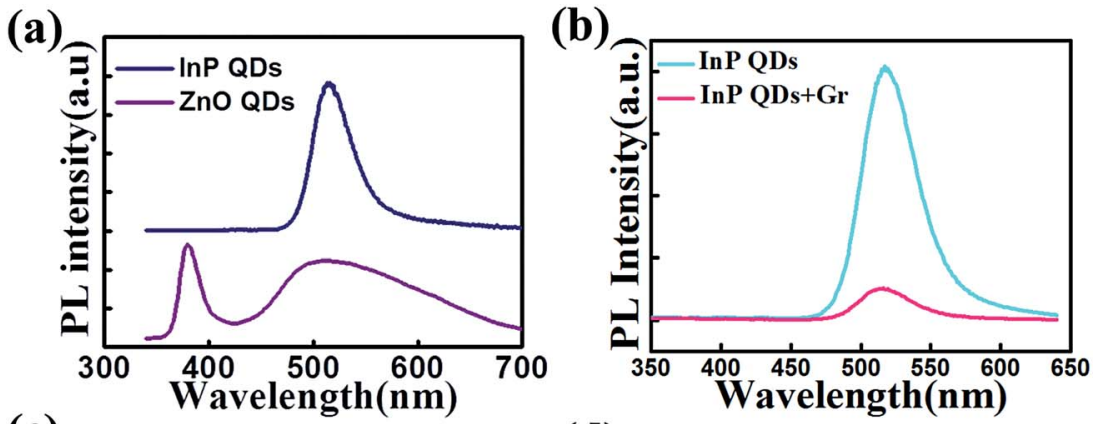

(c)

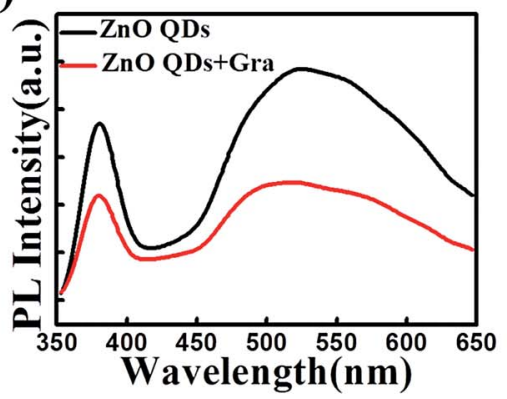

(d)
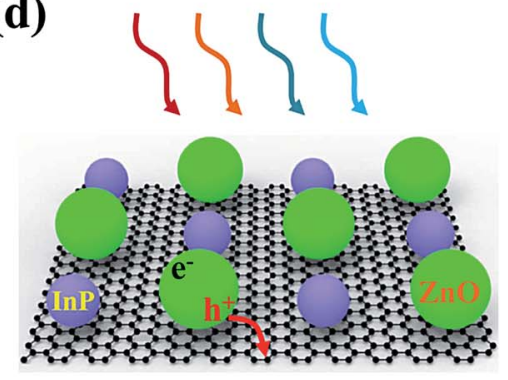

Fig. 4 (a) $\mathrm{PL}$ spectra of $\mathrm{InP}$ and $\mathrm{ZnO}$ QDs in solvent. $\mathrm{PL}$ spectra of the $\operatorname{InP}$ (b) and $\mathrm{ZnO}$ (c) QDs with and without $\mathrm{Gr}$ underlying on the $\mathrm{SiO} / \mathrm{Si}$ substrate using $325 \mathrm{~nm} \mathrm{He}-\mathrm{Cd}$ laser as the excitation source. (d) Physical picture of InP and ZnO QDs generated photo-induced p-doping in Gr.

Based on thermionic-emission theory, saturation current density $J_{0}$ can be described as eqn (2):

$$
J_{0}=A T^{2} \exp \frac{-q \phi}{K T}
$$

where $K$ is the Boltzmann constant, $N_{\mathrm{IF}}$ is the junction ideality factor, $q$ is the value of electron charge, $T$ is the absolute temperature ( $T=293 \mathrm{~K}), A$ is the effective Richardson constant of n-type GaAs (8.16 $\mathrm{A} \mathrm{kcm}^{-2}$ ), ${ }^{32}$ and $\phi$ is the junction barrier height related to the differences between the work function of trilayer $\mathrm{Gr}$ and $\mathrm{n}$-GaAs at zero bias voltage. The $N_{\mathrm{IF}}$ values for the device without and with QDs are 2.8, 2.6 and 2.3, respectively, which are larger than ideal factor. This downward trend of $N_{\mathrm{IF}}$ is probably attributed to shrunken values of parallel resistance. ${ }^{27}$ Physical picture of electronic band alignment of QDs/Gr/GaAs heterostructure is shown in Fig. 5(d). GaAs substrate is heavily (a)

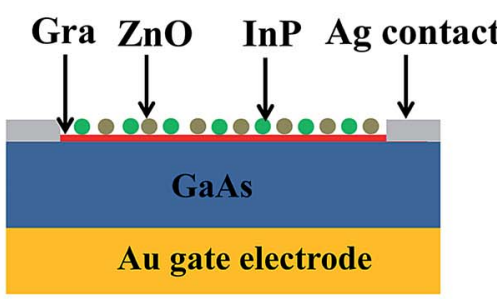

(c)

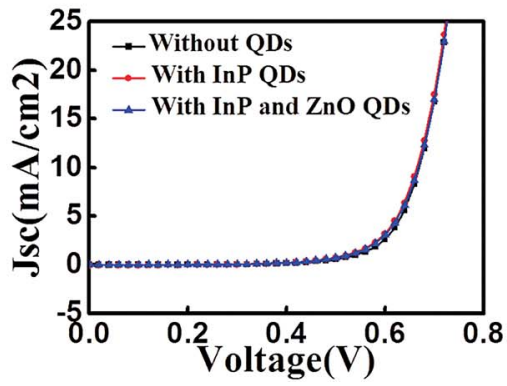

(b)

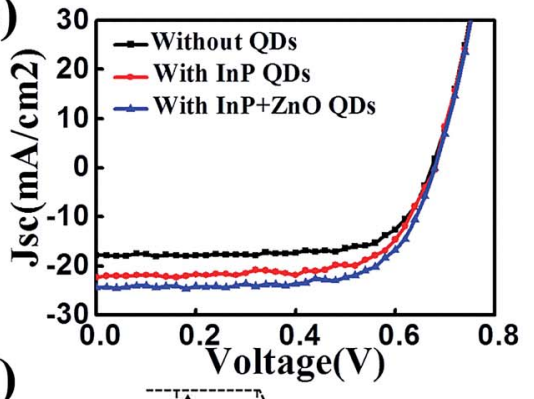

(d)

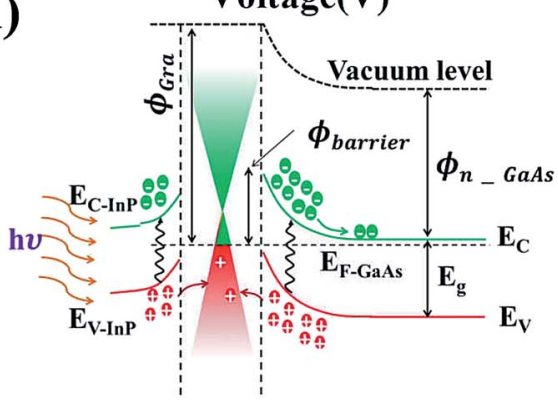

Fig. 5 (a) The schematic cross-section view of Gr/GaAs solar cell with two different kinds of semiconductor QDs. Experimental J-V curves under AM1.5G illumination (b) and in the dark of the solar device with and without InP and ZnO QDs (c). (d) Physical picture of electronic band structure of InP and ZnO QDs coating on Gr/GaAs heterostructure solar cell. 

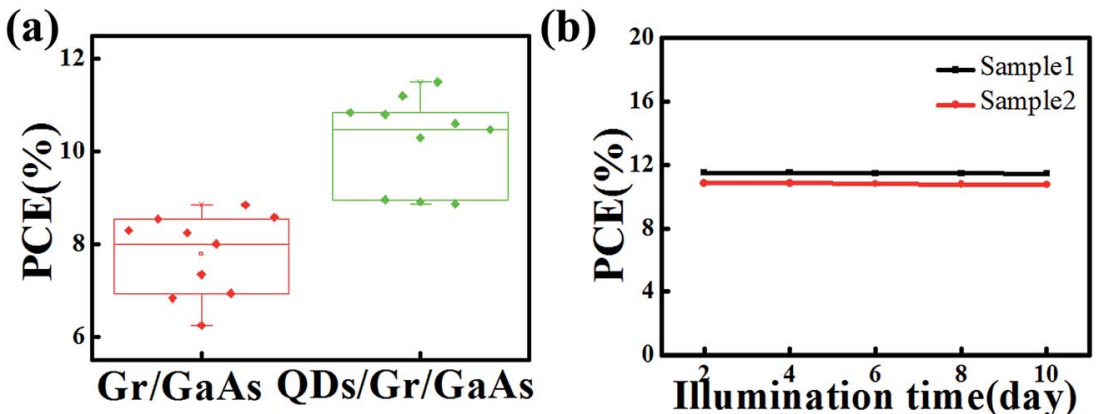

Fig. 6 (a) PCE distribution of the Gr/GaAs and QDs/Gr/GaAs solar cells. (b) Stability of the QDs/Gr/GaAs solar cells under AM1.5G illumination.

n-doped with an electron concentration around $1 \times 10^{18} \mathrm{~cm}^{-3}$. Being heavily n-doped, the work function of GaAs substrate is close to its electron affinity $(4.07 \mathrm{eV}) .{ }^{33}$ The electron affinity of $\mathrm{Gr}$ (the $D$-value of vaccum level and Dirac point) is about $4.6 \mathrm{eV}$ (ref. 34) and its work function is varied depending on the doping concentration. Therefore, a depletion region is formed in the interface of heterostructure by a build-in barrier under van der Waals force due to a clear difference of work function between Gr and GaAs. Without QDs, the light-induced electrons and holes originated in GaAs are collected by GaAs and Gr, respectively. Although the carriers excited by short wavelength photons can be effectively collected, the excess carriers generated by long wavelength can be inefficiently collected owing to several micrometers of diffusion length. In the case can a part of photon be drowned. With some appropriate QDs upper surface of Gr/GaAs solar cell, the photo-induced holes in both GaAs and QDs can inject into $\mathrm{Gr}$ in atomic scale, which raises the collection of holes by $\mathrm{Gr}$ and brings about performance enhancement of solar cells.

Eventually, stability and repeatability of DQs/Gr/GaAs solar cell are well investigated. Statistical analysis of PCE is operated by ten samples of QDs/Gr/GaAs solar cells. Specifically, the PCE distribution of each of the ten samples of Gr/GaAs and QDs/Gr/ GaAs solar cells is illustrated in Fig. 6(a) where the result of obviously enhanced PCE of solar cells can easily be observed after introducing QDs, fully illustrating the practicability of photo-induced doping of Gr/GaAs solar cells. Furthermore, two samples of solar cells with QDs under sunlight illumination for 10 days are randomly used for investigation of stability. As shown in Fig. 6(b), PCE degradation is as low as $0.61 \%$ for these solar cells, indicating photo-induced doping $\mathrm{Gr} / \mathrm{GaAs}$ solar cell with good stability can be sustained operation.

\section{Conclusion}

In conclusion, an ingenious approach to obtain the even higher PCE of Gr/GaAs by making full use of solar energy has been proposed. Two kinds of semiconductor QDs, InP and ZnO QDs, are applied to improve the performance of $\mathrm{Gr} / \mathrm{GaAs}$ solar cell by photo-induced holes doping of Gr. To be specific, the PCE of Gr/ GaAs can be increased from original $8.57 \%$ to $11.50 \%$ after spinning InP and ZnO QDs step by step. Therefore, under this cooperative photo-induced doping, the final PCE is improved by
$34.2 \%$. Good stability of QDs/Gr/GaAs solar cell is also fully confirmed. Further work may be necessary to explore the relation between the different combinations of various QDs that can absorb photon with different energy. Overall, this paper provides an easy and feasible way to develop promising $\mathrm{Gr}$ / semiconductor heterostructure devices for photovoltaic and optoelectronic applications.

\section{Acknowledgements}

S. S. Lin thanks the support from the National Natural Science Foundation of China (No. 51202216, No. 51551203 and No. 51502264), Special Foundation of Young Professor of Zhejiang University (No. 2013QNA5007).

\section{Notes and references}

1 W. Liao, D. Zhao, Y. Yu, C. R. Grice, C. Wang, A. J. Cimaroli, P. Schulz, W. Meng, K. Zhu, R.-G. Xiong and Y. Yan, Adv. Mater., 2016, 28, 9333-9340.

2 L. M. Pazos-Outón, M. Szumilo, R. Lamboll, J. M. Richter, M. Crespo-Quesada, M. Abdi-Jalebi, H. J. Beeson, M. Vrućinić, M. Alsari, H. J. Snaith, B. Ehrler, R. H. Friend and F. Deschler, Science, 2016, 351, 1430-1433.

3 M. Saliba, T. Matsui, K. Domanski, J.-Y. Seo, A. Ummadisingu, S. M. Zakeeruddin, J.-P. Correa-Baena, W. R. Tress, A. Abate, A. Hagfeldt and M. Grätzel, Science, 2016, 354, 206-209.

4 F. De Nicola, M. Salvato, C. Cirillo, M. Crivellari, M. Boscardin, M. Scarselli, F. Nanni, I. Cacciotti, M. D. Crescenzi and P. Castrucci, Carbon, 2016, 101, 226234.

5 K. P. Loh, S. W. Tong and J. S. Wu, J. Am. Chem. Soc., 2016, 138, 1095-1102.

6 T. Salim, H. W. Lee, L. H. Wong, J. H. Oh, Z. A. Bao and Y. M. Lam, Adv. Funct. Mater., 2016, 26, 51-65.

7 K. H. Hendriks, A. S. G. Wijpkema, J. J. van Franeker, M. M. Wienk and R. A. J. Janssen, J. Am. Chem. Soc., 2016, 138, 10026-10031.

8 Y. Guo, Y. Li, O. Awartani, J. Zhao, H. Han, H. Ade, D. Zhao and H. Yan, Adv. Mater., 2016, 28, 8483-8489.

9 C. Z. Li, J. Huang, H. X. Ju, Y. Zang, J. Y. Zhang, J. F. Zhu, H. Z. Chen and A. K. Y. Jen, Adv. Mater., 2016, 28, 7269-7275. 
10 D. P. McMeekin, G. Sadoughi, W. Rehman, G. E. Eperon, M. Saliba, M. T. Hörantner, A. Haghighirad, N. Sakai, L. Korte, B. Rech, M. B. Johnston, L. M. Herz and H. J. Snaith, Science, 2016, 351, 151-155.

11 K. A. Bush, C. D. Bailie, Y. Chen, A. R. Bowring, W. Wang, W. Ma, T. Leijtens, F. Moghadam and M. D. McGehee, $A d v$. Mater., 2016, 28, 3937-3943.

12 J. Liu, S. Lu, L. Zhu, X. Li and W. C. H. Choy, Nanoscale, 2016, 8, 3638-3646.

13 X. Q. Li, S. S. Lin, X. Lin, Z. J. Xu, P. Wang, S. J. Zhang, H. K. Zhong, W. L. Xu, Z. Q. Wu and W. Fang, opt. Express, 2016, 24, 134-145.

14 A. K. Geim, Science, 2009, 324, 1530-1534.

15 R. R. Nair, P. Blake, A. N. Grigorenko, K. S. Novoselov, T. J. Booth, T. Stauber, N. M. R. Peres and A. K. Geim, Science, 2008, 320, 1308.

16 S. Chen, Z. Han, M. M. Elahi, K. M. M. Habib, L. Wang, B. Wen, Y. Gao, T. Taniguchi, K. Watanabe, J. Hone, A. W. Ghosh and C. R. Dean, Science, 2016, 353, 1522-1525.

17 K. S. Novoselov, Z. Jiang, Y. Zhang, S. V. Morozov, H. L. Stormer, U. Zeitler, J. C. Maan, G. S. Boebinger, P. Kim and A. K. Geim, Science, 2007, 315, 1379.

18 A. A. Balandin, S. Ghosh, W. Z. Bao, I. Calizo, D. Teweldebrhan, F. Miao and C. N. Lau, Nano Lett., 2008, 8, 902-907.

19 C. Lee, X. D. Wei, J. W. Kysar and J. Hone, Science, 2008, 321, 385-388.

20 S. J. Zhang, S. S. Lin, X. Q. Li, X. Y. Liu, H. A. Wu, W. L. Xu, P. Wang, Z. Q. Wu, H. K. Zhong and Z. J. Xu, Nanoscale, 2016, 8, 226-232.

21 X. L. Zhang, C. Hagglund and E. M. J. Johansson, Adv. Funct. Mater., 2016, 26, 1253-1260.
22 X. Q. Li, W. C. Chen, S. J. Zhang, Z. Q. Wu, P. Wang, Z. J. Xu, H. S. Chen, W. Y. Yin, H. K. Zhong and S. S. Lin, Nano Energy, 2015, 16, 310-319.

23 D. L. Boyda, V. V. Braguta, S. N. Valgushev, M. I. Polikarpov and M. V. Ulybyshev, Phys. Rev. B: Condens. Matter Mater. Phys., 2014, 89, 6.

24 D. M. Forrester and F. V. Kusmartsev, Nanoscale, 2014, 6, 7594-7603.

25 P. H. Cheng, L. G. Wu, X. Li, T. Li, H. X. Zhao and J. L. Bao, Opt. Commun., 2015, 335, 279-283.

26 S. S. Lin, Z. Q. Wu, X. Q. Li, Y. J. Zhang, S. J. Zhang, R. Panneerselvam and J. F. Li, Adv. Energy Mater., 2016, 6, 5.

27 S. S. Lin, X. Q. Li, S. J. Zhang, P. Wang, Z. J. Xu, H. K. Zhong, Z. Q. Wu and H. S. Chen, Appl. Phys. Lett., 2015, 107, 5.

28 P. Wang, S. S. Lin, G. Q. Ding, X. Q. Li, Z. Q. Wu, S. J. Zhang, Z. J. Xu, S. Xu, Y. H. Lu, W. L. Xu and Z. Y. Zheng, Appl. Phys. Lett., 2016, 108, 4.

29 N. Petrone, C. R. Dean, I. Meric, A. M. van der Zande, P. Y. Huang, L. Wang, D. Muller, K. L. Shepard and J. Hone, Nano Lett., 2012, 12, 2751-2756.

30 X. S. Li, W. W. Cai, J. H. An, S. Kim, J. Nah, D. X. Yang, R. Piner, A. Velamakanni, I. Jung, E. Tutuc, S. K. Banerjee, L. Colombo and R. S. Ruoff, Science, 2009, 324, 1312-1314.

31 G. Konstantatos, M. Badioli, L. Gaudreau, J. Osmond, M. Bernechea, F. P. G. de Arquer, F. Gatti and F. H. L. Koppens, Nat. Nanotechnol., 2012, 7, 363-368.

32 M. S. Carpenter, M. R. Melloch, M. S. Lundstrom and S. P. Tobin, Appl. Phys. Lett., 1988, 52, 2157-2159.

33 W. J. Jie, F. G. Zheng and J. H. Hao, Appl. Phys. Lett., 2013, 103, 4.

34 S. Sarkar, E. Bekyarova and R. C. Haddon, Acc. Chem. Res., 2012, 45, 673-682. 